T. B. age 22. Admitted Nov. 8, 1919. Patient has had good eyes up till 18 mo. ago, when the right eye became painful. It has been irritated most of the time, until quite recently, when the irritation subsided. The sight in this eye has gradually failed, so that on admission, she has only perception of hand movements at two feet. The left eye became inflamed two months previous to admission, with pain, photophobia and lacrimation.

Fxamination.-Right eye showed atrophy of eyeball with diminished tension. Cornea clear with lower half of anterior chamber filled with a grayish exudate. Iris dull, atrophic and adherent to lens. Pupillary space occluded by a membrane.
Left eye showed circumcorneal injection with a bluish zone of deep hyperemia. Cornea clear, and on Descemet's membrane were the small mutton fat deposits. Iris hyperemic and swollen, and adherent to lens in places by fresh exudates. The lens itself was clear but opacities were seen in the vitrcous. Fundus indistinct, but apparently normal. Vision 20/200.

In this case a blood Wassermann was negative. Infected tonsils were removed, and an injection of $0.5 \mathrm{mg}$. $O$. T. gave a positive focal and general reaction. Tuberculin was started in therapeutic doses and is still being given, with moderate slow improvement in the left eye.

\title{
REFERENCES.
}

1. Fuchs: "Text Book of Ophthalmology."

2. Jackson: Journal American Medical Association, Feb. 14, 1920.

\section{INTRADURAL TUMORS OF OPTIC NERVE WITH REPORT OF A CASE}

\author{
Ernest Scott, B.S., M.D., and Frank F. Schmidt, B.S., \\ COLUMBUS, oHro.
}

Cases of this condition are worthy of record both for their rarity and their clinical interest. With the report of such a case, studied in the Department of Pathology of the Ohio State University, there is here included a brief statement of our present knowledge regarding such tumors.

In discussing intradural tumors of the optic nerve Parsons gives a brief historical sketch, in which he states that Wishart in 1833 and Middlemore in 1838 were the first to report such cases and that Goldzieher in 1873 separated optic nerve tumors from the extradural orbital tumors. von Graefe in 1864 gave the main diagnostic points of such tumors, while Leber, in 1877, was the first to attempt the differentiation of tumors arising within the sheath of the nerve from those without. In 1901 Byers collected one hundred and two cases from the literature, which he subdivided upon a histologic basis. The most recent study of this class of tumors has been made by Hudson, who in 1912 collected one hundred and fifty-four cases. These he divided into three groups: (1) Gliomatosis,
(2) fibromatosis of the nerve sheath, and (3) endothelial tumors of the nerve sheath, placing one hundred and eighteen of the cases in the first group and again subdividing this group into. those designated as (a) gliomatosis, (b) probably gliomatosis, and (c) probably not gliomatosis. Under the second heading he placed six cases, the remaining cases falling under endothelial tumors of the sheath.

Such primary intradural tumors are met with most commonly during the earlier years of life, corresponding in this respect with the gliomatous tumors of the brain. Eighty-five of the cases cited by Byers gave exact data as to age; of these sixty-seven occurred in children before the fifteenth year, fifty per cent of the sixty-seven occurring before the fifth year, only four of 
this series occurring subsequent to the twenty-fifth year of life. Because of this early age incidence and of the absence of any definite etiologic factor, it has become generally accepted that these tumors usually arise from some form of embryonal or congenital defect in the structure of the nerve tissue. In some instances, however, direct trau$m a$ is given as the exciting cause.

The usual symptoms presented by patients suffering from intradural tumors of the optic nerve are a slowly progressing exophthalmus which, in

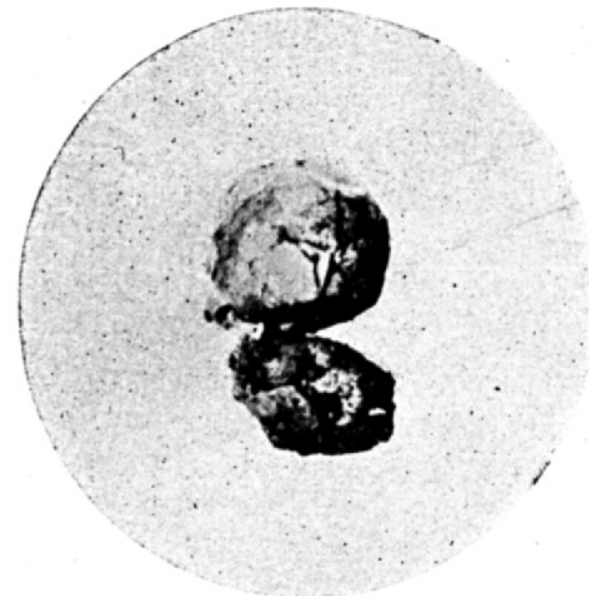

Fig. 1.-Photograph of eye with tumor of the
nerve attached.

the majority of instances, is directly forward, and a gradually developing blindness. Movements of the eyeball are limited ard pain is absent until the tumor has attained a considerable size. The ophthalmoscopic examination reveals an optic neuritis and a choked disc.

Anatomically, the tumors are situated within the dural sheath of the optic nerve and are sharply differentiated, both macroscopically and microscopically, from tumors arising within the sheath itself or growing in the orbit outside the sheath The intradural tumors differ greatly in size, varying from a barely perceptible swelling in the course of the nerve to tumors 3 centimeters in diameter. The anterior portion of the nerve, or that portion in immediate proximity to the eyeball, is usually not involved, the main enlargement being found in the middle portion of the nerve; it may, however, involve that portion in the posterior part of the orbit and even extend into the intracranial portion of the nerve. The growths also vary markedly in consistency, some being quite firm and dense, others being soft and of a more gelatinous consistency. Ewing describes the structure of gliomas occurring in the brain substance, as consisting of "glia cells and glia fibers of various forms and in varying proportions. In many the typical spider cell is pre-

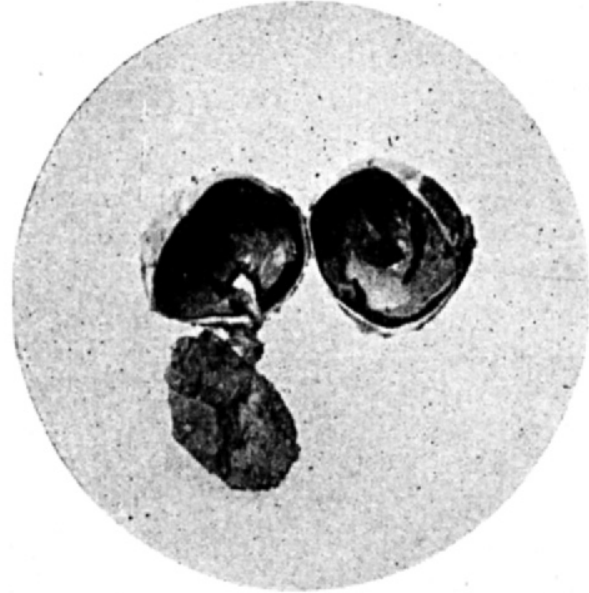

Fig. 2.-Photogiaph of eyeball after section with the
tumor of the nerve.

dominant. This cell has a small compact nucleus and a scanty cytoplasm, from which radiate numerous comparatively short fibers. In other cases the cells are larger and the fibers longer and less numerous. In certain tumors the cells are large and possess cytoplasmic processes containing large nuclei or multiple nuclei, and closely resemble ganglion cells." Tumors growing within the optic nerve, grouped under the head of. gliomata, differ rather markedly from this description. These tumors contain the same small cell with dense nucleus and scanty cytoplasm. The cells, however, are rather widely. separated in a substance that is spoken of as gelatinous or edematous and in which fibrils are made out with some difficulty, and with certainty only after special neuroglia stains have been used. 
Histologically, the structure of intradural tumors has given rise to considerable discussion. Byers states that "such tumors do not correspond with any special type of growth, but there is constantly present in the same specimen several phases of developing connective tissue." He has drawn an analogy between these changes and the changes occurring in elephantiasis due to primary obstruction of the lymph flow and uses the term "fibromatosis" to define such growths. He further states that there is essentially an over-

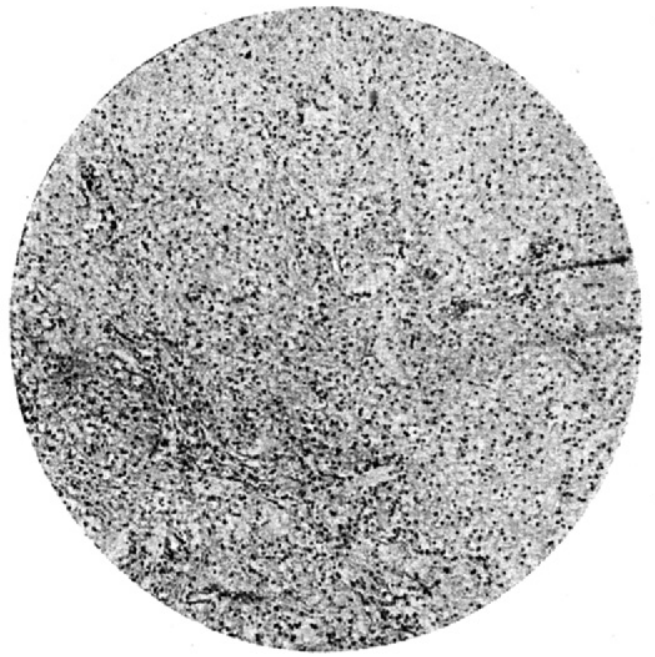

Fig. 3,-Pholomicrograph showing general structure of the tunor. $16 \mathrm{~mm}$. objective.

growth of connective tissue, which is protean in character, and that the feature described as myxomatous is in reality a simple edema due to elephantiasis. Ewing, in discussing two cases examined by himself and which he designates "neurofibroma of the optic nerve," states that they "consist of numerous small cells of the type of the glia cell, which lie in compact groups or singly in a mucous matrix." Hudson suggests the term "degenerative gliomatosis," implying a generalized overgrowth of neuroglial tissue of infiltrating character, dependent on some degenerative change of unknown etiology.

Again, the exact origin of the cells composing these tumors, whether epiblastic or mesoblastic, has also caused more or less discussion. According to
Prentiss, "the embryologic derivatior. of such tissue is from cells of the netral type, which divide by mitosis and give rise to ependymal cells of the ependymal zone and to indifferent cells of the mantle layer; from the latter cells spongioblasts and neuroblasts are formed. The spongioblasts are transformed into neuroglia cells and fibers which form the supporting tissue of the nervous system, while the neuroblasts of the primitive cells, by developing cell processes, are converted into true neurons." With this view Mallory

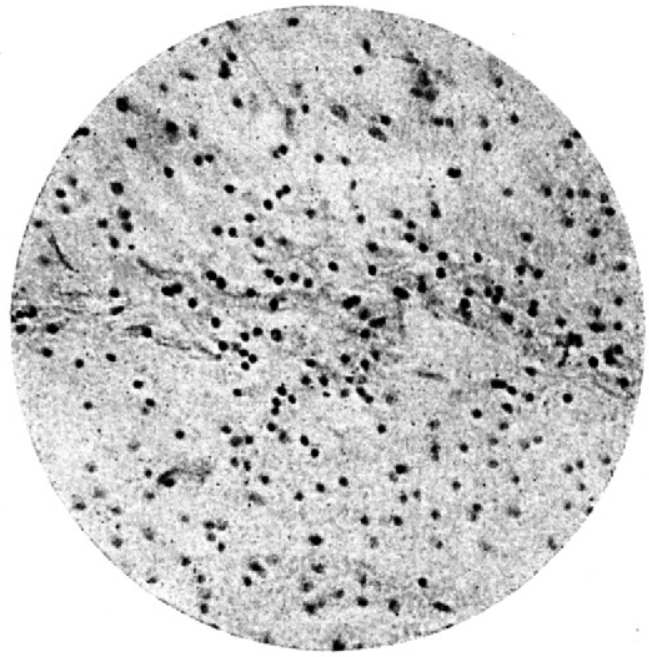

Fig. 4.-Photomicrograph illustrating the two types of glia cells with the fibrillar network. $4 \mathrm{~mm}$. objective.

coincides, describing a "glioblastoma as a tumor of epiblastic origin in which the cells tend to differentiate into neuroglia." Hatai, on the other hand, has proven with some conclusiveness that a certain portion of the neuroglial cells are derived from the mesoblastic tissue which enters the nervous system about the blood vessels and that the neuroglia cells, with larger nuclei and less abundant fibrils, are of true mesoblastic origin.

From this brief discussion of the more general features of such cases, it may be seen that primary intradural tumors of the optic nerve are worthy of record not alone for their rarity, but also for their clinical interest and because they represent rather complex histopathologic features. The following case is therefore reported because 
of the definite interest that obtains in this subject.

CASE Report.-A boy, age 10, white, had been brought to a physician because of a gradual loss of sight of the right eye. There had been no unusual disease of the part and no history of previous injury to the eye or cranium. At this time there was no exophthalmus. The boy had had the usual diseases of childhood; otherwise the history is negative. Seven and a half months later he came under the observation of an ophthalmologist. At this time exophthalmus was pronounced, the eye being forced directly forward; blindness was complete. Immediate surgical removal of the eye was advised and consented to by the parents.

Gross Examination. The tumor consists of a mass $3 \times 2.5 \mathrm{~cm}$., whose closest proximity to the globe is $8 \mathrm{~mm}$. The tumor is somewhat irregular and shows distinct nodules on its surface. It is intimately surrounded by a rather dense fibrous capsule, which undoubtedly is the dural sheath. Upon section, the tissue is of a grayish color, which in some areas has a reddish tinge. It varies in consistency in different portions, some areas being dense, others softer, altho in no portion could it be described as edematous or gelatinous. The optic nerve, at its entrance into the eye ball, is normal in appearance and measures $3 \mathrm{~mm}$. in diameter. At a point some $6 \mathrm{~mm}$. from the globe the nerve is slightly constricted and beyond this point becomes lost in the anterior border of the tumor mass.

Microscopic examination shows that the tumor consists of a loose feltwork of fibrillar tissue, in which are scattered numerous small, deeply stained nuclei, which show a rather definite tendency to group themselves. The cytoplasm of these cells is scanty and, in many instances, cannot be distin- guished. In some areas pale staining, round or oval nuclei are seen scattered about among the more typical glia cells. These cells are considered as belonging to the second group of glia cells mentioned by Hatai. Extending thru the mass are strands of coarse fibrous tissue, which subdivide the tumor into irregular areas. The blood vessels are numerous and pass directly thru the substance of the tumor, being enclosed by a connective tissue sheath, which is intimately surrounded by the fibrillar material. In certain areas no fibrils can be distinguished, the substance appearing more or less homogeneous. In this homogeneous matrix are spaces, of irregular size, resembling spaces in which fluid had accumulated, and giving the impression that the tissue had been more or less edematous in character.

The sections of the tumor were stained with hematoxylin-eosin, Mallory's phosphotungstic acid hematoxylin, Van Giesen's alum-hematoxylin, and Pal-Weigert stains. The Pal-Weigert stain was not successful, due to improper fixing of the tissues before reaching the laboratory. The phosphotungstic acid hematoxlin, howcver, showed distinctly the neuroglia fibrils, while the hematoxylin-eosin and hematoxylin-Van Gieson stains permit nicely the demonstration of the general structure of the tumor.

Conclusion.-From a perusal of the literature and the careful study of the tumor presented in this paper, it appears to the writers that the growth here recorded should be classed as a true tumor and that the terms "degenerative" and "fibrosis" are not adequate to explain its existence.

We wish to acknowledge our indebtedness to Dr. R. L. Barnes for the specimen and to Dr. R. Blee Smith for the clinical history of the case. .

\section{BIBLIOGRAPHY.}

1. Byers: Studies from the Royal Victoria Hospital, Montreal, 1901.

2. Byers: Tumors of the Optic Nerve. Jour. Am. Med. Assn., Vol. 63, pp. 20-25.

3. Ewing: Neoplastic Diseases, p. 418.

4. Hatai, S.: Ill. Med. Jour., 1902-1903, Series 4.

5. Hudson: Royal London 'Ophth, Hosp. Report. London, 1912, XVIII, pt.' 3, pp. 317-459.

6. Mallory: Principles of Pathologic Histology, p. 348 .

7. Parsons: Pathology of the Eye, Vol. 2, page 693.

8. Prentiss: Text Book of Embryology, page 302 . 